\title{
Energy-based wear law for oblique impacts in dry environment
}

Thibaut Souilliart ${ }^{a, b}$, Emmanuel Rigaud ${ }^{a}$, Alain Le Bot ${ }^{a}$, Christian Phalippou ${ }^{b}$

${ }^{a}$ Laboratoire de Tribologie et Dynamique des Systèmes, UMR CNRS 5513, Ecole Centrale de Lyon, Université de Lyon, 69134, Ecully, France

${ }^{b}$ Commissariat à l'Energie Atomique et aux Energies Alternatives CEA-Saclay, DEN, DANS, DM2S, SEMT, DYN, F-91191 Gif-sur-Yvette, France

\section{Abstract}

Wear generated by repetitive impacts between an Inconel tube sample and a stainless steel flat bar target is studied through experiments in dry environment. Incident energy and angle of impacts are controlled, normal and tangential loads during impact are measured as well as rebound energy and angle of impacts. Impacts characteristics are analyzed and the influence of the energy loss during impacts and the incidence angle on generated wear is analyzed. Wear volume is found to be proportional to the energy loss, for which a maximum is observed at an incidence angle close to $25^{\circ}$ from horizontal. An impact wear energy coefficient is introduced and an impact wear law with a strong mechanical meaning is proposed. This energy-based law well predicts the observed experimental wear.

\section{Highlights}

Impact wear between an Inconel tube and a stainless steel bar is studied.

Wear is observed to be proportional to the energy loss during impacts.

The energy loss during impacts strongly depends on incidence angle.

An energy-based impact wear law is proposed.

\section{Keywords}

Impact wear

Dry friction

Energy model

Wear model 
Nomenclature

\begin{tabular}{|c|c|}
\hline$c_{S}$ & Piezoelectric sensor damping \\
\hline$c_{c}$ & Contact damping \\
\hline$d_{i m p}$ & Contact duration \\
\hline$e_{n}$ & Restitution coefficient of impact \\
\hline$f$ & Excitation frequency \\
\hline$f_{0}$ & First natural frequency of the projectile \\
\hline$f_{c}$ & Frequency of the projectile mass $m$ oscillating on the contact stiffness $k_{c}$ \\
\hline$f_{S}$ & Internal resonance frequency of the impact wear test machine \\
\hline$k$ & Stiffness of the projectile \\
\hline$k_{a}$ & Surface asperities crushing stiffness \\
\hline$k_{c}$ & Contact stiffness \\
\hline$k_{f t}$ & Force transducer stiffness \\
\hline$k_{h}$ & Hertzian contact stiffness \\
\hline$k_{S}$ & Piezoelectric sensor stiffness \\
\hline$k_{t}$ & Tube ovalisation stiffness \\
\hline$l$ & Mean experimental value of apparent contact length \\
\hline$m$ & Projectile mass \\
\hline$m_{s}$ & Mass supported by force transducer \\
\hline$t$ & Time \\
\hline$t_{i n} ; t_{i t}$ & Time location of normal/tangential load beginning during impact \\
\hline$t_{r n} ; t_{r t}$ & Time location of normal/tangential load end during impact \\
\hline$v_{r} ; v_{r n} ; v_{r t}$ & Rebound velocity; Normal/Tangential component of rebound velocity \\
\hline$v_{i} ; v_{i n} ; v_{i t}$ & Incident velocity; Normal/Tangential component of incident velocity \\
\hline$D_{s}$ & Normal displacement of piezoelectric sensor \\
\hline$D_{n} ; D_{t}$ & Normal/Tangential component of displacement \\
\hline$E^{*}$ & Equivalent Young modulus in Hertz theory \\
\hline$F_{n} ; F_{t}$ & Normal/Tangential component of load \\
\hline$K$ & Impact wear energy coefficient \\
\hline$N$ & Number of impacts during a complete impact wear test \\
\hline$P_{n} ; P_{t}$ & Normal/Tangential impulse during impact \\
\hline$R^{*}$ & Equivalent radius in Hertz theory \\
\hline$T_{i}$ & Incident energy \\
\hline$T_{L} ; T_{L n} ; T_{L t}$ & Energy loss; Normal/Tangential component of energy loss \\
\hline$T_{L}^{*}$ & Normalized energy loss \\
\hline$V^{-} ; V^{+}$ & Negative/Positive wear volume \\
\hline$V_{i m p}$ & Wear volume per impact \\
\hline$\alpha_{i}$ & Incidence angle of the projectile \\
\hline$\alpha_{r}$ & Rebound angle of the projectile \\
\hline$\beta$ & Asperity radius of curvature \\
\hline$\lambda$ & Particle shape coefficient \\
\hline$\mu$ & Impulse ratio \\
\hline$\mu_{c}$ & Critical impulse ratio \\
\hline
\end{tabular}




\section{Introduction}

Repetitive impacts between Steam Generator (SG) tubes and Anti-Vibration Bars (AVB) in Pressurized Water Reactors (PWR) can lead to substantial wear over time. When wear is too large, costly maintenance operations are required. Thus, understanding the relationship between impacts characteristics and wear is of great interest.

Impacts lead to several types of wear according to the motions and the bodies involved [1]. Specifically, two main categories of impacts exist whether it involves important incident mass and low velocity (percussive impact) or low mass and large velocity (particle erosion). Consequently, two very different categories of wear models are proposed. Concerning percussive impact wear, Engel [2-4] develops a model for which wear formation depends on surfaces conformance and wear time evolution depends on shear stress. Levy [5] proposes a model for which wear is proportional to load and sliding distance during impact. Connors [6], Frick [7] and Hoffman [8] also propose impact wear models derived from Archard equation. The wear law proposed by Lewis includes both a term derived from Engel model and a dependency with sliding distance [9]. Gessesse [10] and Attia [11] extend the delamination theory of sliding wear from Suh [12] to percussive impact wear, with a specific interest to the contact geometry at the asperity scale. Concerning erosion wear, three types of models can be distinguished [13]. Finnie [14,15] and Bitter [16] propose models for a rigid grain cutting into a ductile metal. Hutchings [17], Follansbee [18], Ratner [19] and Sundararajan [20] develop fatigue models which involve a critical accumulated strain required to generate wear. Jahanmir [21] extends the delamination theory of sliding wear from Suh [12] to erosion wear. The atomistic simulations of Aghababaei [22] lead to a better understanding of wear mechanisms in general, and of impact wear in particular.

Impact characteristics are deeply studied by Stronge [23]. The analysis of velocities, kinetic energy, forces, friction, stick and slip regions of the contact during impact results in a rich but complex formulation of impact characteristics. Brach [24,25] uses a classical impulse and momentum theory to express these characteristics, especially the energy loss during an impact. It leads to simpler and more intelligible formulations with a high degree of physical meaning. Brach [25] observes a good correlation between the energy loss during an impact and erosive wear results from literature. No comparison is carried out between this model and percussive impact wear observations.

A lot of experimental studies have been carried out in the last decades about impact wear in nuclear field. Guinot [26] and Zaghdoudi [27] have listed many impact test machines and gather them into two categories whether or not priority is given to reproducing real PWR environment. Ko [28], Cha [29] and Blevins [30] among others studied impacts and wear with real environment test machines. Sorokin [31], Rice [32] and Pick [33] developed analytical test machines to study normal impact only. These test machines are expected to have better characteristics than the ones which reproduce real environment but the precision of the dynamics control is very different from one apparatus to another. A lack of analytical experimental apparatus with a large range of possible incidence angles is to be noted. 
In the present work, wear of a SG tube sample subjected to repetitive impacts against an AVB sample is studied. Section 1 presents the experimental apparatus that has been designed and used. Section 2 presents a description of the impacts based on the time evolution of normal and tangential displacements and loads during them. Section 3 presents the impacts characteristics experimentally obtained: restitution coefficient, impulse ratio and energy loss. Section 4 presents an energy-based analysis of generated wear and introduces an energy-based impact wear law.

\section{Experimental apparatus and method}

\subsection{Impact wear test machine}

The experiment aims to analytically study impact wear between a SG tube sample and an AVB sample. The principle of the experiment is the following. The SG tube sample is repetitively thrown against the AVB sample with a controlled incidence energy and angle. At the end of the experiment, wear mainly observed on the SG tube sample is measured. The experimental set-up is shown in Fig. 1.

The impact wear test machine consists of an excitation system, a projectile, and a target. The target is composed of the AVB sample maintained by a holder. The latter is fixed to a force transducer. The set of sample, holder, and force transducer is designed to be very stiff. The projectile is composed of the SG tube sample fixed to a holder. A loading mass is attached to the holder (total mass of the projectile: $m=0.12 \mathrm{~kg}$ ). The projectile is connected to two shakers placed at $\pm 45^{\circ}$ to the vertical by two identical springs (stiffness $k=590 \mathrm{~N} / \mathrm{m}$ ). The natural frequency $f_{0}$ of the impactor (projectile on its springs) is $f_{0} \cong 10 \mathrm{~Hz}$. The projectile is attached at the extremity of a horizontal flexible beam (not represented in Fig. 1). This beam being stiff in the $X$-direction (traction-compression) and compliant in the YZ-directions (flexion), the movement of the projectile is enforced to stay in the YZ-plane.

The trajectory of the SG tube sample is controlled using the two shakers. The shakers apply to the SG tube sample two orthogonal forces which can be controlled independently. The desired value of incident energy is obtained by controlling the amplitude of the sinusoidal inputs. The incidence angle

$\alpha_{i}$ to the horizontal is obtained by setting a particular phase difference between the two inputs. Any impact with an incident energy from $10^{-3} \mathrm{~mJ}$ to $2 \mathrm{~mJ}$ and an incidence angle between $5^{\circ}$ and $90^{\circ}$ can be performed.

\subsection{Signal measurement}

The normal and the tangential contact loads are measured during impacts using a 3-axis piezoelectric force transducer fixed to the AVB sample. The force transducer stiffness is equal to 4500 $\mathrm{N} / \mu \mathrm{m}$ and its sensitivity is $-3.8 \mathrm{pC} / \mathrm{N}$ for normal axis. Its stiffness is equal to $700 \mathrm{~N} / \mu \mathrm{m}$ and its sensitivity is $-8 \mathrm{pC} / \mathrm{N}$ for tangential axis. Signals are acquired using a dynamic acquisition card with a high sampling rate $(50 \mathrm{kHz})$ in order to correctly measure the contact load time evolution during impacts. 
The trajectory of the SG tube sample in the plane of motion is measured by two laser displacement sensors. The measurement range is $\pm 10 \mathrm{~mm}$, the linearity equals to $\pm 0.02 \%$ of the full scale and the repeatability is $0.025 \mu \mathrm{m}$.

A complete time acquisition of contact loads and displacements during the full length of the test is impossible due to storage space limitations. Therefore, signals are acquired during 2000 evenly distributed acquisition time windows of 1 second.

\subsection{Materials properties}

The experiments focus on the wear of an Inconel 690 SG tube sample against a 410s steel flat AVB sample. Tables 1 and 2 present the composition and mechanical properties of the materials.

\begin{tabular}{|c|c|c|c|c|c|c|c|c|}
\hline \multirow[t]{2}{*}{ Composition (\%) } & $\mathrm{Ni}$ & $\mathrm{Cr}$ & $\mathrm{Fe}$ & C & $\mathrm{Si}$ & $\mathrm{Mn}$ & $S$ & $\mathrm{Cu}$ \\
\hline & 58 & $27-31$ & $7-11$ & $<0.05$ & $<0.5$ & $<0.5$ & $<0.015$ & $<0.5$ \\
\hline Elastic modulus (GPa) & 207 & & & & & & & \\
\hline Shear modulus (GPa) & 79 & & & & & & & \\
\hline Yield stress (MPa) & 379 & & & & & & & \\
\hline
\end{tabular}

Table 1. Composition and mechanical properties of Inconel 690 SG tube sample

\begin{tabular}{|c|c|c|c|c|c|c|}
\hline \multirow[t]{2}{*}{ Composition (\%) } & C & Si & $\mathrm{Mn}$ & $\mathrm{P}$ & $\mathrm{S}$ & $\mathrm{Cr}$ \\
\hline & $<0.08$ & $<1$ & $<1$ & $<0.04$ & $<0.015$ & $12-14$ \\
\hline Elastic modulus (GPa) & \multicolumn{6}{|l|}{205} \\
\hline Shear modulus (GPa) & \multicolumn{6}{|l|}{80} \\
\hline Yield stress (MPa) & \multicolumn{6}{|l|}{290} \\
\hline
\end{tabular}

Table 2. Composition and mechanical properties of 410s steel AVB sample

\subsection{Wear volume analysis}

At the end of a test, the worn SG tube sample is removed from the test rig and wear scars are analyzed with an optical interferometer. Negative, positive and natural volumes of wear are then retrieved. The uncertainty on wear volume is estimated with the interferometer characteristics and the surface adjustment. Minimal wear volume that can be measured is $10^{-3} \mathrm{~mm}^{3}$.

\subsection{Testing parameters}

The influence of the incidence angle is studied in the range $5^{\circ}-85^{\circ}$. During each test, the incidence angle of impacts $\alpha_{i}$ is controlled to be constant. The impacting incident kinetic energy is controlled to be equal to $0.55 \mathrm{~mJ}$, corresponding to an incident velocity of $70 \mathrm{~mm} / \mathrm{s}$. The test duration is $20 \mathrm{~h}$ and the excitation frequency $f$ is $20 \mathrm{~Hz}$ so that the total number of impacts is about 1.4 million. The tests 
are carried out at ambient temperature in dry condition. A total of 21 tests are performed at different incidence angles. They give a good confidence in the repeatability of the results.

\section{Description of individual impact}

\subsection{Time evolution of displacements}

Fig. 2 displays the trajectory of the SG tube sample in the plane of motion. Fig. 3 displays large scale time evolution of normal and tangential displacements during several typical impacts and their short scale time evolution during one particular impact. An impact is defined as application of substantial mechanical actions during a very small period of time. It is characterized by a sudden variation of load and velocity. In particular, an inversion of normal velocity of the SG tube sample is observed after the impact in Fig. 2. These figures show that the trajectory is fairly controlled so that an almost periodic behavior is observed.

For each impact, a normal restitution coefficient $e_{n}$ is identified from measured normal velocity immediately before and after the impact:

$$
e_{n}=-\frac{v_{r n}}{v_{i n}}
$$

But $v_{i n}$ and $v_{r n}$ can be expressed as functions of respectively $\alpha_{i}$ and $v_{i}$ and $\alpha_{r}$ and $v_{r}$ (see Fig. 1 for definition of the angles $\alpha_{i}$ and $\alpha_{r}$ ):

$$
\begin{aligned}
& v_{i n}=v_{i} \sin \alpha_{i} \\
& v_{r n}=v_{r} \sin \alpha_{r}
\end{aligned}
$$

Substituting Eqs. (2) and (3) into Eq. (1) gives:

$$
e_{n}=-\frac{v_{r} \sin \alpha_{r}}{v_{i} \sin \alpha_{i}}
$$

Since an impact always dissipates energy, the ratio between rebound velocity $v_{r}$ and incident velocity $v_{i}$ can never exceed 1 . Nevertheless, we observe that rebound angle $\alpha_{r}$ can be higher than incident angle $\alpha_{i}$ and therefore $v_{r n}$ can be higher than $v_{i n}$. This may lead to a restitution coefficient $e_{n}$ greater than 1 without violating the energy conservation. It is especially the case for grazing incidence $\left(\alpha_{i} \ll 1\right)$.

\subsection{Time evolution of loads}

Fig. 4 displays large scale time evolution of normal and tangential loads during several typical impacts and their short scale time evolution during one particular impact. The contact loads are very similar in shape and amplitude from one impact to another, highlighting once again a quasi-periodic behavior. On a single impact scale, two phases are observed: a contact phase and a free flight phase. 
During the contact phase, the time evolution of normal load has a bell shape like expected for a typical shock [2]. First, surfaces approach each other and normal load increases to a maximum (compression period). Then, surfaces move away and normal load decreases to zero when the contact ceases (rebound period). The shock duration is defined as the duration of application of normal load and usually ranges from 0.35 to $0.5 \mathrm{~ms}$. The slight perturbation of the bell shape near the maximal value is due to the influence of the compliance of the target on the measurements. This perturbation is studied in section 2.5 in order to verify that its influence on the measured contact force is weak, especially on the values of maximal load and impact duration.

During the free flight phase, the normal load oscillates around zero with a frequency equal to $6.5 \mathrm{kHz}$. This is the consequence of the free vibration of the target after the impact.

The obliqueness of impacts leads to the existence of a tangential component of load during impact whose time evolution can also be observed in Fig. 4. Sliding, sticking, and rolling can occur during the contact depending on incidence characteristics and contact properties $[25,34,35]$. While sliding and sticking lead to the existence of a tangential load component, tangential load during rolling is imperceptible. Thus, the dates of start and end of tangential load application $\left(t_{i t}\right.$ and $\left.t_{r t}\right)$ can differ from those of normal component $\left(t_{i n}\right.$ and $\left.t_{r n}\right)$. Tangential impact duration is defined as the duration of application of tangential load.

For each impact, the normal and tangential impulses are defined by the variation of momentums during the impact:

$$
\begin{gathered}
P_{t}=\int_{t_{i t}}^{t_{r t}} F_{t}(t) d t=m\left(v_{r t}-v_{i t}\right) \\
P_{n}=\int_{t_{i n}}^{t_{r n}} F_{n}(t) d t=m\left(v_{r n}-v_{i n}\right)
\end{gathered}
$$

and an impulse ratio $\mu$ is introduced:

$$
\mu=\frac{P_{t}}{P_{n}}
$$

Note that Eqs. (5) and (6) lead to two different methods to compute the impulse ratio. The first one is based on the difference of incident and rebound velocities while the second one is obtained by time-integration of the force signals. Both methods give similar results.

\subsection{Energy loss during impacts}


The energy loss is an important impact characteristic commonly mentioned in the literature on wear [2,14,16,36-39]. The balance of energy between incident kinetic energy, energy loss, and rebound kinetic energy is expressed by:

$$
T_{i}=\frac{1}{2} m\left(v_{i n}^{2}+v_{i t}^{2}\right)=\frac{1}{2} m\left(v_{r n}^{2}+v_{r t}^{2}\right)+T_{L}
$$

In this equation, the rotational energy is supposed to be negligible. Eqs. (1-3) and (5-7) give rebound velocities as a function of incidence angle $\alpha_{i}$, restitution coefficient $e_{n}$, and impulse ratio $\mu$. This leads to the following expression of dimensionless energy loss.

$$
T_{L}^{*}\left(\alpha_{i}, e_{n}, \mu\right)=T_{L} / T_{i}=\sin ^{2} \alpha_{i}\left(1+e_{n}\right)\left[1-e_{n}+\frac{2 \mu}{\tan \alpha_{i}}-\mu^{2}\left(1+e_{n}\right)\right]
$$

The energy loss $T_{L}^{*}$ may be separated in two terms: $T_{L n}^{*}$ corresponds to the loss of energy associated to normal motion and $T_{L t}^{*}$ corresponds to the energy loss due to tangential effects. The normal loss of energy is defined by:

$$
T_{L n}^{*}=\frac{1}{2} m\left(v_{i n}^{2}-v_{r n}^{2}\right) / T_{i}=\sin ^{2} \alpha_{i}\left(1-e_{n}^{2}\right)
$$

where the second equality stems from Eqs. (1) and (2). This also corresponds to $T_{L n}^{*}$ at the special value $\mu=0$ as it may be seen from Eq. (9).

The tangential loss of energy is defined by:

$$
T_{L t}^{*}=\frac{1}{2} m\left(v_{i t}^{2}-v_{r t}^{2}\right) / T_{i}=\sin ^{2} \alpha_{i}\left(1+e_{n}\right)\left[\frac{2 \mu}{\tan \alpha_{i}}-\mu^{2}\left(1+e_{n}\right)\right]
$$

Of course, the total energy loss $T_{L}^{*}$ is the sum of normal loss $T_{L n}^{*}$ and tangential loss $T_{L t}^{*}$.

\subsection{Dynamic model of the test apparatus}

Considering only the normal direction, the test apparatus can be described using a two degrees of freedom model as proposed in $[40,41]$. The projectile is modeled by a mass $m$ at position $D_{n}$. The contact is modeled by a damping factor $c_{c}$ and a stiffness $k_{c}$. The target is modeled by a mass $m_{s}$, a damping factor $c_{s}$ and a stiffness $k_{s}$ which is associated to the target compliance, especially to the piezoelectric transducer characteristics. Other parts of the target are assumed to be infinitely rigid bodies.

The impact is divided into two phases: the contact phase during which the mass $m$ oscillates on the system \{contact + target\}, and the free flight phase during which the mass $m_{s}$ oscillates on the target stiffness $k_{s}$. The equations of motion are:

$$
\left\{\begin{array}{l}
m \ddot{D_{n}}+k_{c} D_{n}-k_{c} D_{s}=-c_{c} \dot{D_{n}}+c_{c} \dot{D_{s}} \\
m_{s} \ddot{D_{s}}+\left(k_{s}+k_{c}\right) D_{s}-k_{c} D_{n}=-c_{s} \dot{D_{s}}
\end{array}\right.
$$

during the contact phase and 


$$
\left\{\begin{array}{c}
m \ddot{D_{n}}=0 \\
m_{s} \ddot{D_{s}}+k_{s} D_{s}=-c_{s} \dot{D_{s}}
\end{array}\right.
$$

during the free flight phase.

Masses of Eqs. (12) and (13) are weighed with a balance. The other coefficients of Eqs. (12) and (13) are determined using an experimental modal analysis. Two complementary approaches have been used: analysis of the free vibratory response to a shock hammer and analysis of the forced vibratory response to a sinusoidal sweep excitation. The numerical values of these coefficients are summarized in Table 3:

\begin{tabular}{|r|r|r|r|r|r|r|}
\hline$c_{c}(\mathrm{~kg} / \mathrm{s})$ & $c_{s}(\mathrm{~kg} / \mathrm{s})$ & \multicolumn{1}{|l|}{$k_{c}(\mathrm{~N} / \mathrm{m})$} & $k_{s}(\mathrm{~N} / \mathrm{m})$ & $m(\mathrm{~kg})$ & $m_{s}(\mathrm{~kg})$ \\
\hline 5000 & & 10 & $9.810^{6}$ & $2.510^{9}$ & 0.12 & 1.52 \\
\hline
\end{tabular}

Table 3. Values of parameters of the dynamic model

The stiffness $k_{c}$ is associated to the combination of the Hertzian contact stiffness $k_{h}$, the tube ovalisation stiffness $k_{t}$ and the surface asperities crushing stiffness $k_{a}$. In the present case of a cylinder in contact with a plane, the Hertzian contact stiffness $k_{h}$ is given by:

$$
k_{h}=\frac{\pi l E^{*}}{4}=3.610^{8} \mathrm{~N} / \mathrm{m}
$$

with $l \approx 4 \mathrm{~mm}$ and $E^{*} \approx 115 \mathrm{GPa}$.

The tube ovalisation stiffness $k_{t}$ is calculated numerically using a finite element model of the tube held by the tube holder. The displacements at the bottom of the tube are blocked and a normal load of $50 \mathrm{~N}$ is applied to the top of the tube. The tube ovalisation stiffness $k_{t}$ is obtained from the maximal tube displacement observed: $k_{t}=1.210^{8} \mathrm{~N} / \mathrm{m}$. The surface asperities crushing stiffness $k_{a}$ is calculated by considering the Hertzian elastic contact between two spheres with an equivalent radius $R^{*}$ equal to a typical value of asperity radius of curvature $\beta \approx 100 \mu \mathrm{m}$ :

$$
k_{a}=2\left(\frac{3}{4} R^{*} E^{* 2} F_{n}\right)^{1 / 3}=7.310^{6} \mathrm{~N} / \mathrm{m}
$$

where $E^{*} \approx 115 \mathrm{GPa}$ and $F_{n} \approx 50 \mathrm{~N}$.

The surface asperities crushing stiffness $k_{a}$ is much lower than the others contact stiffness. It can be deduced that the interface is governed by contact asperities. To verify this hypothesis, the measured contact duration observed on force signal may be compared with the duration of an impact $d_{\text {imp }}$ calculated in the case of an elastic impact between a moving mass $m$ with velocity $v_{i}$ and a target considered as two spheres with a typical value of asperity radius of curvature $\beta \approx 100 \mu \mathrm{m}$ ([42]):

$$
d_{i m p}=2.87 \frac{m^{2 / 5}}{\left(\beta E^{*^{2}} v_{i}\right)^{1 / 5}}
$$

Since $m \approx 0.12 \mathrm{~kg}, E^{*} \approx 115 \mathrm{GPa}$ and $v_{i} \approx 0.07 \mathrm{~m} / \mathrm{s}$, we obtain $d_{\text {imp }} \approx 0.49 \mathrm{~ms}$ in a fine agreement with experimental values. This observation also highlights that contact is controlled by 
dynamic effects of elastic forces during the impact and not by a quasi-static contact like it would be obtained with a hydraulic press instead of the two shakers. During impacts, the influence of external forces applied by shakers remains weak.

The numerical value of the target stiffness $k_{s}$ is in the same order of magnitude of the manufacturer's value of force transducer stiffness $k_{f t} \approx 4.510^{9} \mathrm{~N} / \mathrm{m}$. Therefore, the compliance of the target mainly corresponds to the force transducer characteristics.

\subsection{Comparison between experimental impacts and dynamic model}

Eqs. (12) and (13) are solved using measured incident normal velocity as initial condition. Modeled time evolution of $D_{n}$ (normal SG tube displacement) and $D_{S}$ (normal piezoelectric sensor displacement) are obtained by a numerical simulation.

In Fig. 6 are shown the time evolution of the reaction force $k_{s} D_{s}$ (as measured by the sensor) simulated by the present model, the measured signal as delivered by the actual sensor and the contact force $k_{c} D_{n}$ simulated by the model. Since $k_{c}<k_{s}$, the contact duration is mainly controlled by the half-period $\frac{T}{2}$ of the projectile mass $m$ oscillating on the contact stiffness $k_{c}$, with:

$$
\frac{1}{T}=f_{c}=\frac{1}{2 \pi} \sqrt{\frac{k_{c}}{m}} \cong 1.4 \mathrm{kHz}
$$

with $k_{c}=9.810^{6} \mathrm{~N} / \mathrm{m}$ and $m=0.12 \mathrm{~kg}$.

After the impact, the oscillation in force signal corresponds to the free vibration of the sensor with a frequency $f_{s}$ :

$$
f_{s}=\frac{1}{2 \pi} \sqrt{\frac{k_{s}}{m_{s}}} \cong 6.5 \mathrm{kHz}
$$

with $k_{s}=2.510^{9} \mathrm{~N} / \mathrm{m}$ and $m_{s}=1.52 \mathrm{~kg}$. Free oscillations on time evolution of tangential load have a lower frequency.

A good correlation is found between the time evolution of experimental and modeled normal load which shows that the proposed model is relevant. The influence of the eigenfrequency $f_{s}$ on the evolution of load during impact is highlighted: measured value of reaction force is about $10 \%$ higher than the contact force. However, no significant difference is observed concerning the impact duration. A similar reasoning is used for tangential component and no significant difference is observed on tangential maximal load and impact duration either. 


\section{Statistical analysis of impacts}

\subsection{Restitution coefficient of impact}

Fig. 7 displays normal restitution coefficient $e_{n}$ versus incidence angle $\alpha_{i}$ for data measured during 21 complete tests of $20 \mathrm{~h}$. The coefficient $e_{n}$ is calculated from measured normal incident and rebound velocities using Eq. (1). Each color corresponds to a particular test. Each point of the cloud corresponds to a single impact. Data from 40000 impacts are shown for each test, corresponding to the impacts measured during the 2000 evenly distributed time acquisition windows. The range of abscissa values is divided in ten equal parts. Error bars are estimated for each part and correspond to standard deviation of abscissa for horizontal bars and ordinate for vertical bars.

It can be observed on Fig. 7 that mean value of $e_{n}$ decreases from 0.9 to 0.6 when incidence angle increases from $0^{\circ}$ to $45^{\circ}$. Experimental values of $e_{n}$ are rather much dispersed in this range. Furthermore, $e_{n}$ increases from 0.6 to 0.8 when angle increases from $45^{\circ}$ to $90^{\circ}$. A similar order of magnitude has been observed in the literature [43] for cylinder-cylinder contact in the same incident velocity range. The dashed black line corresponds to a $2^{\text {nd }}$ order polynomial fit defined by:

$$
e_{n}\left(\alpha_{i}\right)=1.610^{-4} \alpha_{i}^{2}-1.510^{-2} \alpha_{i}+0.95
$$

where $\alpha_{i}$ is in degrees.

\subsection{Friction during impact}

Friction is analyzed through the impulse ratio $\mu$ defined in Eq. (7). By analyzing the mechanical problem of a single rigid projectile impacting a massive flat surface, Brach [25] introduced a critical impulse ratio $\mu_{c}$ controlling the transition from sliding throughout the impact to sliding and rolling during the contact phase. This critical ratio is given by:

$$
\mu_{c}=\frac{1}{1+\lambda} \frac{1}{1+e_{n}} \frac{1}{\tan \alpha_{i}}
$$

The critical impulse ratio $\mu_{c}$ depends on normal restitution coefficient $e_{n}$, incidence angle $\alpha_{i}$ and a shape factor $\lambda$ related to the projectile shape. The analysis of the contact stiffness in section 2.4 shows that the interface is governed by the contact asperities. Thus, the projectile has the shape of the surface asperity into contact. Therefore, $\lambda$ is chosen equal to zero as contact asperities can be considered as point masses.

For any impact conditions, $\mu$ is always lower or equal to $\mu_{c}$. If $\mu<\mu_{c}$, sliding occurs throughout the impact and consequently $\mu$ corresponds to the friction coefficient. This case occurs when incidence angles are low. Beyond an incidence angle threshold, $\mu=\mu_{c}$ and both sliding and rolling occur during the contact phase.

Fig. 8 shows measured impulse ratio $\mu$ versus incidence angle $\alpha_{i}$. The experimental values of $\mu$ are obtained from normal and tangential impulses using Eq. (7). Normal and tangential impulses are 
calculated by integrating measured normal and tangential loads during the shock duration using Eq. (5) and (6). A plateau is observed in the range $0^{\circ}-30^{\circ}$ for which $\mu \approx 1$. In the range $30^{\circ}-90^{\circ}, \mu$ decreases with increasing incidence angle as tangential impulse is becoming lower, up to $\mu=0$ for normal impacts.

The solid black line corresponds to:

$$
\mu\left(\alpha_{i}\right)=\left\{\begin{aligned}
1, & 0^{\circ} \leq \alpha_{i} \leq 35^{\circ} \\
\mu_{c}\left(\alpha_{i}\right), & \alpha_{i} \geq 35^{\circ}
\end{aligned}\right.
$$

where $\mu_{c}\left(\alpha_{i}\right)$ is calculated from Eq. (20) with $\lambda=0$ and $e_{n}=0.7$. The experimental impulse ratio is well predicted by $\mu_{c}$ on the range $30^{\circ}-90^{\circ}$. According to the Brach theory, sliding and rolling should therefore occur during these impacts. This prediction of impulse ratio $\mu$ evolution versus incidence angle explains previous observations made without interpretation in References $[44,45]$. For the range $0^{\circ}-30^{\circ}$, the plateau $\mu \approx 1$ is also consistent with the Brach model and corresponds to sliding throughout the impact.

\subsection{Energy loss during impacts}

The experimental study of the impact characteristics in sections 3.1 and 3.2 reveals a dependence between impulse ratio $\mu$ and incidence angle $\alpha_{i}$ by Eq. (21), and between restitution coefficient $e_{n}$ and incidence angle $\alpha_{i}$ by Eq. (19). But general expressions of energy losses $T_{L}^{*}, T_{L n}^{*}$, and $T_{L t}^{*}$ in terms of $\alpha_{i}, e_{n}$, and $\mu$ have been obtained in Eqs. (9), (10), and (11). Therefore, $T_{L}^{*}, T_{L n}^{*}$, and $T_{L t}^{*}$ can be expressed as a function of $\alpha_{i}$ only by a simple substitution.

Fig. 9 shows normal dimensionless energy loss $T_{L n}^{*}$ during impact versus incidence angle $\alpha_{i} . T_{L n}^{*}$ is calculated from measured incident and rebound normal velocities using Eq. (10) for each impact. Normal dimensionless energy loss increases with impact incidence from 0 for low angles to 0.6 at $70^{\circ}$. Then, it decreases to 0.3 for normal impacts. Fig. 10 shows tangential dimensionless energy loss $T_{L t}^{*}$ during impact versus incidence angle $\alpha_{i} . T_{L t}^{*}$ is calculated from measured incident and rebound tangential velocities using Eq. (11). Tangential energy loss is equal to zero for incidence angles $0^{\circ}$ and $90^{\circ}$. A maximum of energy loss is observed at $25^{\circ}$ from horizontal and is equal to 0.7 . Fig. 11 shows total dimensionless energy loss $T_{L}^{*}$ during impact versus incidence angle $\alpha_{i}$. Total energy loss $T_{L}^{*}$ corresponds to the sum of $T_{L n}^{*}$ and $T_{L t}^{*}$. $T_{L}^{*}$ is equal to zero for incidence angle $0^{\circ}$. A maximum equal to 0.9 is observed at $30^{\circ}$.

The energy losses defined by Eqs. (9), (10) and (11) are calculated with the modeled values of $e_{n}$ and $\mu$ from Eqs. (19) and (21), and are shown with a solid black curve on each figure. A good correlation between experimental results and the Brach formulation of energy loss confirms the relevance of the chosen approach to predict the energy loss during an impact. According to these experimental results, it is interesting to note that this approach seems to be verified not only in the case of erosion as initially verified by Brach [25] but also in the case of impact between massive solids. 


\section{Energy-based analysis of wear}

\subsection{Wear scars morphology}

Wear has been observed on both SG tube samples and AVB samples for all incidence angles. But, it has been observed that AVB wear volume is much lower than SG tube wear volume. So only SG tube wear is considered for the rest of this study. Negative volumes of SG tube wear range from $2.10^{6} \mu \mathrm{m}^{3}$ to $12.10^{6} \mathrm{~mm}^{3}$ for the largest wear. A typical wear scar observed by scanning electron microscopy and interfometry is shown in Fig. 12. The shape of the scar is typical of wear induced by a cylinder-plane contact with imperfect parallelism. In this case, the tube is slightly tilted so that the area corresponding to the bottom of the image hits the AVB sample first. Related wear profile is shown in Fig. 13.

A certain amount of positive volume is observed at the peripheral region of the scar. It corresponds to wear debris which reamalgamate with the surface or plastic deformation. These two origins of positive volume are distinguished using interferometry and scanning electron microscope (SEM) techniques. The shape of wear debris is granular and oxides are observed around them $\left(V_{1}^{+}\right.$and

$V_{2}^{+}$in Fig. 13). Plastic deformation is noticed by the existence of a smooth pile in the immediate vicinity of the negative volume $\left(V_{3}^{+}\right.$in Fig. 13). Wear debris are observable more frequently for low angles whereas plastic deformation is observed to happen for larger impact angles.

\subsection{Experimental results on wear}

Several authors observed that wear volume is proportional to the energy loss [2,14,16,36-39]. According to the dependence of energy loss to incident angle highlighted in the previous section, the wear volume is expected to be strongly dependent on incidence angle. This dependence on incidence angle has also been experimentally observed previously $[41,46]$.

Fig. 14 displays experimental wear volume per impact versus average incidence angle for each test. The 21 tests performed are represented by dots. Horizontal error bars display the dispersion of the controlled incidence angle and correspond to the $20^{\text {th }}$ and the $80^{\text {th }}$ percentiles. Wear increases with incidence angle from 0 to $8 \mu \mathrm{m}^{3}$ per impact between $0^{\circ}$ and $25^{\circ}$. A maximum of wear is reached at $25^{\circ}$ from horizontal which is consistent with previous observations $[44,45,47]$. In the range $25^{\circ}-90^{\circ}$, wear decreases with incidence from $8 \mu \mathrm{m}^{3}$ to $3 \mu \mathrm{m}^{3}$ per impact. It should be noted that wear measured at normal incidence is not null contrary to previous observations $[44,45]$.

In Fig. 14, experimental wear volume is compared to the loss of energy per impact: normal component in broken line (corresponding to Fig. 9), tangential component in dotted line (corresponding to Fig. 10) and total loss in solid line (corresponding to Fig. 11). Wear evolution against incidence angle is well predicted by the total energy curve for all the incidence angles. Two cases are observed: for small incidence angles $\left(\alpha_{i} \leq 50^{\circ}\right)$, the tangential component of energy dominates, for larger incidence angles $\left(\alpha_{i} \geq 50^{\circ}\right)$, the normal component of energy dominates. For both cases, a very fine agreement is observed between wear and the total energy loss. This shows that there is no 
preponderant influence of tangential effects over normal effects on wear, which is in contradiction with many studies on impact wear $[45,48]$.

Assuming that the total wear volume $V^{-}$is proportional to the impacts incident energy and to the number of impacts $N$ occurred during a complete test, an impact wear law and an impact wear energy coefficient $K$ are introduced:

$$
\frac{V^{-}}{N T_{i}}=K T_{L}^{*}\left(\alpha_{i}, e_{n}\left(\alpha_{i}\right), \mu\left(\alpha_{i}\right)\right)
$$

where $T_{L}^{*}$ is given in Eq. (9), $e_{n}\left(\alpha_{i}\right)$ in Eq. (19) and $\mu\left(\alpha_{i}\right)$ in Eq. (21).The experimental value of the impact wear energy coefficient $K$ obtained is:

$$
K=1.3410^{4} \mu \mathrm{m}^{3} / \mathrm{J}
$$

The coefficient $K$ may be interpreted as a wear volume per unit mechanical energy lost during impacts. Although the contact is intermittent (and even rare), this coefficient is analogous to Archard's coefficient in continuous sliding contact wear. The coefficient $K$ also may be compared to energy wear coefficient introduced in fretting wear studies $[49,50]$. This coefficient also corresponds to the wear coefficient commonly used in the nuclear industry [51-53]. The observed value of $K$ is compared to the wear coefficients obtained in [52] with a similar experimental configuration but with an environment considered as more wearing (tests in water at $290^{\circ} \mathrm{C}$ ). These wear coefficients range from $110^{4}$ to 5.5 $10^{4} \mu \mathrm{m}^{3} / \mathrm{J}$, which corresponds to the same order of magnitude of the latter observed value of $K$.

\section{Conclusion}

Wear generated by repetitive impacts between a tube sample and a plane sample with constant incident energy is studied. An impact wear test machine is designed with a significant care in obtaining a very fine control of the impact dynamics and a high quality of measurements. Normal and tangential displacements and loads during impacts are measured and analyzed. Impulse ratio, restitution coefficient and energy loss are calculated from these measurements and compared to incidence angle.

The numerous experiments performed show that the restitution coefficient during impacts slightly depends on incidence angle. The impulse ratio follows a two-parts evolution with incidence angle. This highlights two regimes of impacts whether only sliding occur during the contact phase (for small incidence angle) or sliding and rolling (for large incidence). The latter regime is well predicted by the critical impulse ratio introduced by Brach [25]. Experimental dimensionless energy loss is also well predicted by the Brach formulation. This tends to prove the validity of this model in the case of percussive impacts.

Wear is observed for all the experiments performed and is found to be proportional to the energy loss during impacts. Based on these observations, an energy-based impact wear law is proposed together with an impact wear coefficient. It presents a strong dependence on the energy loss during impacts and on the incidence angle. The experimental values of wear are well predicted by this model. 


\section{References}

[1] Ko PL. Metallic wear - a review with special references to vibration-induced wear in power plant components. Tribol Int 1987;20:66-78. doi:10.1016/0301-679X(87)90092-2.

[2] Engel PA. Impact Wear of Materials. vol. 2. 2nd ed. Elsevier; 1978. doi:10.1115/1.3424343.

[3] Engel PA. Percussive impact wear. A study of repetitively impacting solid components in engineering. Tribol Int 1978;11:169-76. doi:10.1016/0301-679X(78)90002-6.

[4] Engel PA, Lyons TH, Sirico JL. Impact wear model for steel specimens. Wear 1973;23:185-201. doi:10.1016/0043-1648(73)90083-5.

[5] Levy G, Morri J. Impact fretting wear in CO2-based environments. Wear 1985;106:97-138. doi:10.1016/0043-1648(85)90106-1.

[6] Connors HJ. Flow-induced vibration and wear of steam generator tubes. Nucl Technol 1981;vol.55, no:311-31.

[7] Frick TTM. An empirical wear projection technology with steam generator tube applications and relations to work-rate and wear simulations/tests. Flow-Induced Vib Noise 1997;2:275-82.

[8] Hofmann PJ, Schettler T, Steininger DA. PWR steam generator tube fretting and fatigue wear phenomena and correlations. ASME Vib Noise 1992;1:211-36.

[9] Lewis R. A modelling technique for predicting compound impact wear. Wear 2007;262:1516-21. doi:10.1016/j.wear.2007.01.032.

[10] Gessesse YB. On the fretting wear of nuclear power plant heat exchanger tubes using a fracture mechanics approach: theory and verification. Concordia University (Canada), 1997.

[11] Attia H. A generalized fretting wear theory. Tribol Int 2009;42:1380-8. doi:10.1016/j.triboint.2009.04.010.

[12] Suh PN. The delamination theory of wear. Wear 1973;25:111-24. doi:Doi: 10.1016/00431648(73)90125-7.

[13] Shewmon P, Sundararajan G. The Erosion of Metals. Annu Rev Mater Sci 1983;13:301-18. doi:10.1146/annurev.ms.13.080183.001505.

[14] Finnie I. Erosion of surfaces by solid particles. Wear 1960;3:87-103. doi:10.1016/00431648(60)90055-7.

[15] Finnie I. Some observations on the erosion of ductile metals. Wear 1972;19:81-90.

[16] Bitter JGA. A study of erosion phenomena. Wear 1963;6:169-90. doi:10.1016/00431648(63)90073-5.

[17] Hutchings IM. A model for the erosion of metals by spherical particles at normal incidence. Wear 1981;70:269-81. doi:10.1016/0043-1648(81)90347-1. 
[18] Follansbee PS, Sinclair GB, Williams JC. Modelling of low velocity particulate erosion in ductile materials by spherical particles. Wear 1982;74:107-22. doi:10.1016/0043-1648(81)90197-6.

[19] Ratner SB, Styller EE. Characteristics of impact friction and wear of polymeric materials. Wear 1981;73:213-34.

[20] Sundararajan G, Shewmon PGG. A new model for the erosion of metals at normal incidence. Wear 1983;84:237-58. doi:10.1016/0043-1648(83)90266-1.

[21] Jahanmir S, Suh NP. Mechanics of subsurface void nucleation in delamination wear. Wear 1977;44:17-38. doi:10.1016/0043-1648(77)90082-5.

[22] Aghababaei R, Warner DH, Molinari J-F. Critical length scale controls adhesive wear mechanisms. Nat Commun 2016;7:11816. doi:10.1038/ncomms11816.

[23] Stronge WJ. Impact mechanics. Cambridge University Press; 2000.

[24] Brach RM. Classical planar impact theory and the tip impact of a slender rod. Int J Impact Eng 1993;13:21-33. doi:10.1016/0734-743X(93)90106-H.

[25] Brach RM. Impact dynamics with applications to solid particle erosion. Int J Impact Eng 1988;7:37-53. doi:10.1016/0734-743X(88)90011-5.

[26] Guinot J. Etude Bibliographique des Travaux Expérimentaux menés sur l'usure par impacts glissements : influence des principaux paramètres. 1989.

[27] Zaghdoudi S. Analyse des phénomènes d'usure par choc et frottement. Université de Paris VI, 1992.

[28] Ko PL. Heat Exchanger Tube Fretting Wear: Review and Application to Design. J Tribol 1985;107:149. doi:10.1115/1.3261010.

[29] Cha JH, Wambsganss MW, Jendrzejczyk JA. Experimental study on impact/fretting wear in heat exchanger tubes. J Press Vessel Technol 1987;109:265-74. doi:10.1115/1.3264862.

[30] Blevins R. Vibration-Induced Wear of Heat exchanger tubes. J Eng Mater 1985;107.

[31] Sorokin GM, Polyanskaya TA. Machine for the investigations into the impact fatigue wear. Zavod Lab 1970;36:611-2.

[32] Rice SL. Reciprocating impact wear testing apparatus. Wear 1977;45:85-95. doi:10.1016/00431648(77)90104-1.

[33] Pick RJ, Brown K, Plumtree A. Techniques in the study of impact and sliding wear of Zircaloy-4. Wear 1979;52:381-92. doi:10.1016/0043-1648(79)90074-7.

[34] Stronge WJ. Smooth dynamics of oblique impact with friction. Int J Impact Eng 2013;51:36-49. doi:10.1016/j.ijimpeng.2012.08.001.

[35] Maw N, Barber JR, Fawcett JN. The oblique impact of elastic spheres. Wear 1976;38:101-14. doi:10.1016/0043-1648(76)90201-5. 
[36] Hutchings IM. Deformation of metal surfaces by the oblique impact of square plates. Int J Mech Sci 1977;19:45-52. doi:10.1016/0020-7403(77)90015-7.

[37] Neilson JH, Gilchrist A. Erosion by a stream of solid particles. Wear 1968;11:111-22. doi:10.1016/0043-1648(68)90591-7.

[38] Tilly GP. A two stage mechanism of ductile erosion. Wear 1973;23:87-96. doi:10.1016/00431648(73)90044-6.

[39] Sundararajan G. A comprehensive model for the solid particle erosion of ductile materials. Wear 1991;149:111-27. doi:10.1016/0043-1648(91)90368-5.

[40] Kaiser A-L, Le Bot A, Bec S, Langlade C, Vernot J. Influence des paramètres mécaniques sur l'usure d'un contact tube-bague en conditions extrêmes. 17ème Congrès Français de Mécanique, Troyes: 2005, p. 1-6.

[41] Kaiser A-L. Caractérisations tribologiques d'un acier inoxydable $\gamma$ sollicité en impacts-glissement dans un environnement sévère. Ecole Centrale de Lyon, 2005.

[42] Johnson KL. Contact mechanics 1984;19:109-10. doi:10.1016/0301-679X(86)90085-X.

[43] Kadmiri Y, Rigaud E, Perret-Liaudet J, Vary L. Experimental and numerical analysis of automotive gearbox rattle noise. J Sound Vib 2012;331:3144-57. doi:10.1016/j.jsv.2012.02.009.

[44] Souilliart T, Rigaud E, Le Bot A, Phalippou C. Wear induced by stochastic sliding impacts. ASME 2015 Press. Vessel. Piîng Conf. PVP2015, ASME; 2015, p. 1-6. doi:10.1115/PVP2015-45811.

[45] Rigaud E, Le Bot A. Influence of incidence angle on wear induced by sliding impacts. Wear 2013;307:68-74. doi:10.1016/j.wear.2013.07.015.

[46] Messaadi M, Kermouche G, Kapsa P. Numerical and experimental analysis of dynamic oblique impact: Effect of impact angle. Wear 2015;332-333:1028-34. doi:10.1016/j.wear.2015.01.070.

[47] Souilliart T, Rigaud E, Le Bot A, Phalippou C. Wear generated by sliding impacts. Proc. Congrès Français Mécanique, Lyon: 2015, p. 1-9.

[48] Ko PL. The significance of shear and normal force components on tube wear due to fretting and periodic impacting. Wear 1985;106:261-81. doi:10.1016/0043-1648(85)90112-7.

[49] Mohrbacher H, Blanpain B, Celis JP, Roos JR, Stals L, Van Stappen M. Oxidational wear of TiN coatings on tool steel and nitrided tool steel in unlubricated fretting. Wear 1995;188:130-7. doi:10.1016/0043-1648(95)06637-3.

[50] Fouvry S, Liskiewicz T, Kapsa P, Hannel S, Sauger E. An energy description of wear mechanisms and its applications to oscillating sliding contacts. Wear 2003;255:287-98. doi:10.1016/S00431648(03)00117-0.

[51] Ko PL. Wear of Power Plant Components Due to Impact and Sliding. Appl Mech Rev 1997;50:387-411. doi:10.1115/1.3101724.

[52] Phalippou C, Herms E, Ruffet F. PWR steam generator tube and AVB wear under perpendicular 
impacting. ASME Press. Vessel. Pip. Conf. July 14-18, Paris, France: 2013, p. 1-10. doi:10.1115/PVP2013-97263.

[53] Frick T, Sobek T, Reavis J. Overview on the Development and Implementation of Methodologies to compare vibration and wear of steam generators tubes. Proc. ASME Symp. Flow-Induced Vib. Vol. 3, Vib. Heat Exch., 1984, p. 149-61. 
Fig. 1. Diagram of the impact wear test machine (1: Displacement sensors, 2: Shakers, 3: Loading mass, 4: Springs, 5: Tube holder, 6: SG Tube, 7: AVB holder and AVB sample, 8: Force transducer)

Fig. 2. Trajectory of the SG tube sample in the plane of motion. (a) Large scale, during 4 impacts. (b) Short scale, during a single impact (dots: measurement points, broken line: best fit).

Fig. 3. Time evolution of normal (solid red line) and tangential (broken blue line) displacements of the SG tube sample. (a) Large scale, during 4 impacts. (b) Short scale, during a single impact.

Fig. 4. Time evolution of normal (solid red line) and tangential (broken blue line) loads. (a) Large scale, during 4 impacts. (b) Short scale, during a single impact.

Fig. 5. Dynamic model of the impact wear test machine (1: Tube, 2: Contact, 3: Piezoelectric Sensor)

Fig. 6. Time evolution of normal load during an impact: experimental reaction force (-) vs numerical reaction force $k_{s} D_{s}(--)$ vs numerical contact force $k_{c} D_{n}(-$.

Fig. 7. Normal restitution coefficient vs incidence angle. Broken black curve: $2^{\text {nd }}$ order polynomial fit.

Fig. 8. Impulse ratio vs incidence angle. Solid black curve: $\mu_{c}\left(\lambda=0, e_{n}=0.7, \alpha_{i}\right)$.

Fig. 9. Normal dimensionless energy loss $T_{L n}^{*}$ during impact versus incidence angle. Cloud: experimental data; solid black line computed from Eqs. (10) and (19).

Fig. 10. Tangential dimensionless energy loss $T_{L t}^{*}$ versus incidence angle. Cloud: experimental data; solid black line computed from Eqs. (11) and (21).

Fig. 11. Total dimensionless energy loss $T_{L}^{*}$ versus incidence angle. Cloud: experimental data; solid black line computed from Eqs. (9), (19) and (21).

Fig. 12. SEM and interferometry images of a tube wear scar

Fig. 13. Tube wear scar profile

Fig. 14. Wear volume per impact versus incidence angle. Energy loss (-); normal component of energy loss (- -); tangential component of energy loss (-.). 